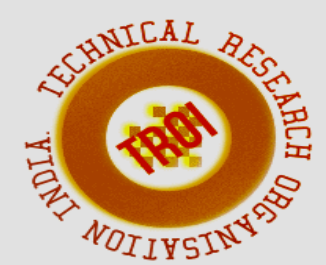

\title{
EXPERIMENTAL INVESTIGATION ON PARTIALLY REPLACEMENT OF BITUMEN WITH WASTE MATERIALS FOR FLEXIBLE PAVEMENT CONSTRUCTION
}

\author{
Teerthananda Sagar C S ${ }^{1}$, Kavitha $V^{2}$, Sultan Fayaz ${ }^{3}$, Ashwini C Goudathi ${ }^{4}$ \\ ${ }^{1,2}$ Assistant Professor, Civil Engineering Dept. - DR.T.TIT, Oorgaum, KGF, Karnataka \\ ${ }^{3,4}$ UG Student, Civil Engineering Dept. - DR.T.TIT, Oorgaum, KGF, Karnataka
}

\begin{abstract}
Roads and highways are among the most important infrastructure systems in the India, traveled on by roughly $65 \%$ of Indians. The conditions of the majority of these roads and highways are considered to be in inferior shape and to provide good all weather road connectivity to unconnected villages, towns and cities are mandatory for the economic development of a country. In spite of increase in blossom pursuits in an evolving nation has led to a swell in population. Due to this the quantity of plastic waste, squandering and tires are increased.
\end{abstract} This is undeniable a genesis for concern squander to disposed by junk yards, landfill antonyms and proclivity, which are perilous and risky. Our aim of the project is to inspect \& study how the waste stuffs will be effectively utilized in construction of surface course and how it acts as additive or modifier which could improve the performance of bitumen properties as bitumen modifier. We have conducted the experiments on the physical properties of bitumen mix by varying from 10 to $60 \%$ of waste plastic (PET), waste rubber, both plastic and rubber equally combined respectively. According to this research we found that $30 \%$ is the optimum percentage of waste stuff can be restored. In addition, the use of these waste substance will play a significant role in reducing the use of non-renewable resources, in constructing sustainable pavements and leads to decrease in cost of construction up to $38.5 \%, 40 \%$ and $39.3 \%$ when waste (plastic, rubber, plastic \& rubber respectively) restored when compare to ordinary bitumen.
Keywords :-( Flexible Pavement, Environmentally Friendly, Marshall Stability, Plastic, Tires, Waste)

\section{INTRODUCTION}

India is a blossoming country where roads and highways are the most important in infrastructure systems. India encompasses a road network and stands second largest road network in the world. As on 31 March 2019.But absence of roads, cars, motorcycles, trucks and plastic materials are almost ineffable. Waste valorization has become central to a more efficient and sustainable development in the India today. The reuse and reinsertion of waste substances in the production chain reduces the consumption of basic commodities and, in many cases, even avert the depletion of valuable natural resources. Moreover, it also alleviates the accumulation of waste at dumpsites, which is now a serious problem. Consequently, great efforts are currently being made to reuse squander stuff in production systems. New technologies can need to be evolved to meet the future demands and needs to decrease the cost of construction. In the construction of flexible pavements, bitumen plays the role of binding the aggregate together by coating over the aggregate. It conjointly helps to boost the strength of the road. But its resistance towards water is poor. Use of plastic and rubber leads to excellent pavement life, driving comfort and low maintenance. This paper aims at proposing a method of disposal of plastic and tire waste by using them on the surface course of the pavement. The Main objective of this study are 
safe and productive disposal of wastes - plastic and tire, study of index properties and suitability of waste bituminous mix on surface course of the pavement and to reduce the cost.

\subsection{Objectives}

1. To investigate the physical properties of bitumen in existence of rubber, PET, both rubber \& PET mixed bitumen at different percentages.

2. To calculate an optimum percentage of waste materials in the bituminous mix.

3. To provide the solution for disposal in a useful way, to reduce the cost of construction and to increase the Marshall Stability value.

\section{LITREATURE REVIEW}

In an endeavor to diminish the ejection of waste stuffs in junk yards and open burning is becoming unacceptable because of rapid depletion of available landfill sites and clear environment. The concept of Sustainable Development, currently a very hot issue, essential that the society as a whole becomes aware of the prerequisite to make the most of all existing resources, trying to minimize creation of residues, a number of models have been presented in the literature. On the other hand, the bitumen modifier development approach has been documented as a better alternative because it overcomes these regression model limitations (S. Rajesekaran and Dr. R. Vasudevan 2013; Athira R Prasad 2015; R. Sujith Kumar 2017; A. Logeshkumaran 2018)

S. Rajesekaran and Dr. R. Vasudevan et al. (2013) conducted research on waste plastics both by domestic and industrial sectors can be used in the production of asphalt

mix. Waste plastic, mainly used for packing are made up of polyethylene, polypropylene, polystyrene this softening varies between $110^{\circ} \mathrm{C}-140^{\circ} \mathrm{C}$ and they do not produce any toxic gases during heating but the softened plastics have tendency to from a film like structure over the aggregate when they sprayed over the hot aggregate@160 $\mathrm{c}$. this PCA bitumen mix showed improved binding property and less wetting property and it also showed higher Marshall stability value in the range of 18$20 \mathrm{KN}$ and load bearing capacity of the road is raised by $100 \%$. Athira R Prasad et al. (2015) It is observed that with increase in bitumen concentration the Marshall stability value increases up to certain bitumen content and there after it decreases. Thus, the maximum stability was obtained at 5\%. The bitumen content corresponding to $4 \%$ air voids was obtained as $5.4 \%$. Hence the Optimum Binder Content was calculated as $5.1 \%$. Voids filled with bitumen should between $75-85$. At 5.1\% bitumen by weight aggregate, VFB was 76.58 which is satisfactory. Flow value corresponding to $5.1 \%$ is 3.4 which is also satisfactory as per standards. It was found that in all three cases, the optimum content was obtained as $6 \%$. Since the Marshall stability is higher in case of PET bottles compared to rubber. R Sujith Kumar et al. (2017) has surveyed on the strength of paving mixes can be enhanced by using various types of modifiers with bitumen such as crump rubber, polypropylene and organic polymers. The use of waste plastic and polypropylene which is used as a modifier by an amount of $1 \%, 3 \%, 5 \%, 7 \%$ by weight of bitumen. Marshal stability test is considered to stimulate with field condition flow and stability of the mix increases after incorporating waste plastic. The stability has been increased by adding $7 \%$ of waste plastic and 5\% of polypropylene. The flow mix decreased by adding $7 \%$ of waste plastic and $5 \%$ of polypropylene when compared to conventional mix. A. Logeshkumaran et al. (2018) inquire on the use of waste material like lignin and plastic as a replacement material for bitumen in the percentage of $5 \%$ \& $10 \%, 10 \%$ \& 15\%, 15\% $\& 20 \%$ and $20 \%$ \& $25 \%$ respectively. It has been found that lignin can act as binding material for asphalt hence improving the properties of bitumen and by partial replacement of lignin and plastic with bitumen a gradual increase is observed at $15 \%$ \& $20 \%$ respectively. From this experimental study it evident that ductility property of the bitumen is increased up to $20 \%$. The stability and flow rate of the partially replaced bitumen as increased up to $5.1 \%$ \& $4.49 \%$ respectively when compared to conventional bitumen specimen. Although this literature review outlines many researchers who have developed models for bitumen modifier, it focused only on projects in 
which conventional bitumen and waste materials were used.

\section{MATERIALS USED}

\subsection{BITUMEN}

Ordinary Bitumen of 50/70 grade is used.

\subsection{COARSE AGGREGATES}

Aggregates which possess sufficient strength, hardness, and toughness. Aggregates specified by IRC.

\subsection{PLASTIC}

PET bottles are in shredded form and they can be used as a binder and/or they can be mixed with a binder like bitumen to enhance their binding property. This may be an honest modifier for the bitumen, used for road construction.

\subsection{RUBBER}

Rubber is actually small pieces of waste tire in shredded form. The rubber never completely dissolves in the bitumen and the product is therefore classed as a nonhomogeneous binder.

\section{METHODOLOGY AND TESTING 4.1 METHODOLOGY}

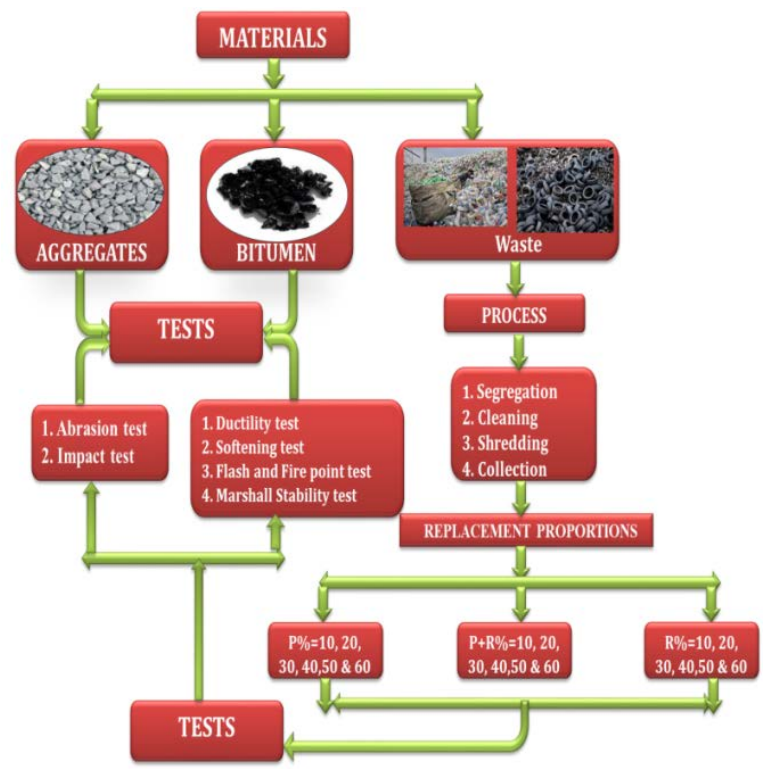

Figure -1: methodology

\subsection{TESTS ON AGGREGATE}

1. Specific Gravity [IS: 2386 (Part 3) 1963].

2. Aggregate Impact Value Test [IS: 2386 (part 4) 1963].

3. Aggregate Abrasion Value [IS: 2386 (Part 4) 1963].
4. Flakiness \& Elongation Index Test [IS: 2386 (part 1) 1963].

\subsection{TESTS ON BITUMEN}

I. Ductility Test [IS: 1208-1978].

II. Ring \& Ball Test (Softening Point) [IS: 1205-1978].

III. Flash and Fire Point [IS 1209-1978].

IV. Marshal Stability Test [MORTH 500].

\section{RESULTS AND DISCUSSION}

\subsection{TESTS ON AGGREGATES}

Aggregates that are taken for the analysis of the construction of pavement are given in below table 5.1 with comparison of standard acceptable values according to their

\begin{tabular}{|c|c|c|c|}
\hline Sl.no & Tests & Results & $\begin{array}{c}\text { Acceptable } \\
\text { value }\end{array}$ \\
\hline 1 & $\begin{array}{l}\text { Specific } \\
\text { Gravity }\end{array}$ & 2.8 & $2.5-3$ \\
\hline 2 & Flakiness & 15 & $<35 \%$ \\
\hline 3 & Elongation & 30.90 & $<35 \%$ \\
\hline 4 & Impact Value & 22.3 & $\begin{array}{l}\text { 10- } \\
\text { 20(Stron } \\
\text { g) } \\
20- \\
\text { 30(Goo } \\
\text { d) }\end{array}$ \\
\hline 5 & $\begin{array}{l}\text { Los-Angeles } \\
\text { Abrasion Test }\end{array}$ & 24.6 & $<30 \%$ \\
\hline
\end{tabular}

particular code specified.

Table 5.1: Test on Aggregates

\subsection{TESTS ON BITUMEN}

Bitumen that is taken for the investigation of the construction of pavement is given in below table 5.2 with comparison of standard acceptable values according to their particular code specified.

Table 5.2: Tests on Bitumen

\begin{tabular}{|c|c|c|c|}
\hline S.no & Tests & Results & $\begin{array}{c}\text { Acceptance } \\
\text { value }\end{array}$ \\
\hline 1. & $\begin{array}{c}\text { Flash And } \\
\text { Fire } \\
\text { Point }\end{array}$ & $\begin{array}{c}180^{\circ} \mathrm{C} \\
210^{\circ} \mathrm{C}\end{array}$ & $\begin{array}{c}220^{\circ} \mathrm{C} \\
\text { (Min) }\end{array}$ \\
\hline
\end{tabular}




\begin{tabular}{|c|c|c|c|}
\hline 2. & $\begin{array}{c}\text { Ring \& } \\
\text { Ball } \\
\text { Test } \\
\text { (Softening } \\
\text { Point) }\end{array}$ & 55 & $47^{\circ} \mathrm{C}($ Min) \\
\hline 3. & Ductility & 54 & $40 \mathrm{~cm}$ (Min) \\
\hline
\end{tabular}

\subsection{TESTS ON WASTE COATED} AGGREGATES

Aggregates that are taken for the research of the construction of pavement are given in below table 5.3 with comparison of standard acceptable values according to IRC specifications.

Table 5.3: Tests on Waste Coated Aggregates

\begin{tabular}{|c|c|c|c|c|}
\hline $\begin{array}{c}\text { Test } \\
\text { on } \\
\text { Aggr } \\
\text { egate } \\
\text { s }\end{array}$ & $\begin{array}{c}\text { Natur } \\
\text { al } \\
\text { Aggr } \\
\text { egate }\end{array}$ & $\begin{array}{c}\text { Plasti } \\
\mathbf{c} \\
\text { coated }\end{array}$ & $\begin{array}{c}\text { Rubb } \\
\text { er } \\
\text { coate } \\
\mathbf{d}\end{array}$ & $\begin{array}{c}\text { (P+R) } \\
\text { coated }\end{array}$ \\
\hline $\begin{array}{c}\text { Los } \\
\text { Angel } \\
\text { es } \\
\text { Abrasi } \\
\text { on test } \\
\text { (\%) }\end{array}$ & 24.6 & 21.15 & 17.54 & $\begin{array}{r}22.0 \\
8\end{array}$ \\
\hline $\begin{array}{c}\text { Impac } \\
\text { t test } \\
\text { (\%) }\end{array}$ & 22.3 & 15.96 & 20.88 & 16.7 \\
\hline
\end{tabular}

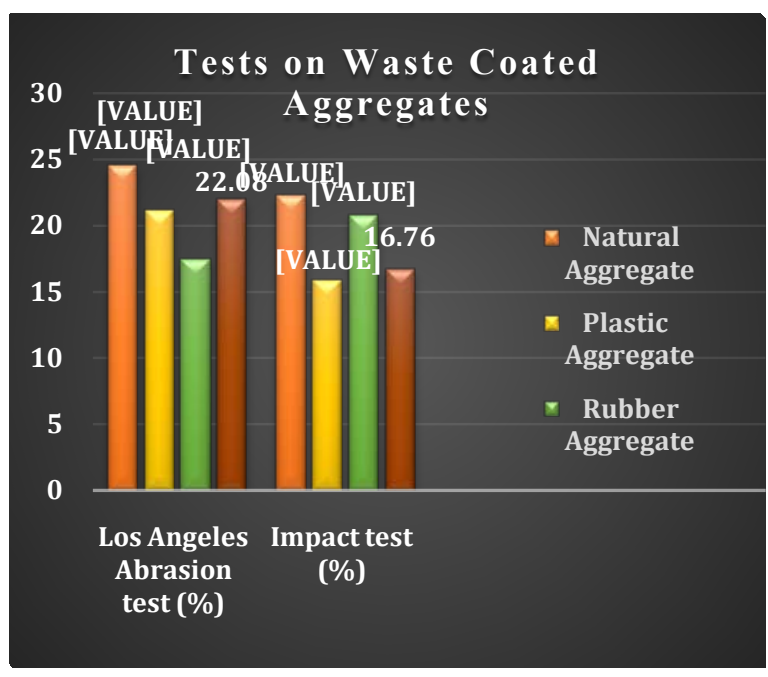

Chart -1: Tests on Waste Coated Aggregates

\subsection{TESTS ON REPLACED BITUMEN}

The bitumen test is conducted and the readings were tabulated. For the readings obtained the following graphs are plotted against Bitumen and various parameters
a) Plastic replacement.
b) Rubber replacement
c) Plastic and rubber replacement

\section{a) PLASTiC REPLACEMENT}

Plastic is restored with bitumen in assorted portions like 10\%, 20\%, 30\%, 40\%,50\% \& 60\% (Marshall Stability test, Ductility test, softening point, flash and fire point test) where execute for every restoration of plastic proportions and note down the readings respectively.

Table 5.4: Tests on Plastic Replaced Bitumen

\begin{tabular}{|c|c|c|c|}
\hline $\begin{array}{c}\text { PLASTIC } \\
\%\end{array}$ & $\begin{array}{c}\text { Ductility } \\
\text { (cm) }\end{array}$ & $\begin{array}{l}\text { Softening } \\
\text { Point } \\
\left({ }^{\circ} \mathbf{C}\right)\end{array}$ & $\begin{array}{c}\text { Flash } \\
\text { and } \\
\text { Fire } \\
\text { Point }\left({ }^{\circ} \mathbf{C}\right)\end{array}$ \\
\hline Bitumen & $54 \mathrm{~cm}$ & $55^{\circ} \mathrm{C}$ & $\begin{array}{c}180^{\circ} \mathrm{C} \\
210^{\circ} \mathrm{C}\end{array}$ \\
\hline 10 & $48 \mathrm{~cm}$ & $53^{\circ} \mathrm{C}$ & $\begin{array}{c}210^{\circ} \mathrm{C} \\
250^{\circ} \mathrm{C}\end{array}$ \\
\hline 20 & $22 \mathrm{~cm}$ & $70^{\circ} \mathrm{C}$ & $\begin{array}{l}212^{\circ} \mathrm{C} \\
259^{\circ} \mathrm{C}\end{array}$ \\
\hline 30 & $14 \mathrm{~cm}$ & $75^{\circ} \mathrm{C}$ & $\begin{array}{l}216^{\circ} \mathrm{C} \\
262^{\circ} \mathrm{C}\end{array}$ \\
\hline 40 & $12.5 \mathrm{~cm}$ & $81^{\circ} \mathrm{C}$ & $\begin{array}{l}220^{\circ} \mathrm{C} \\
265^{\circ} \mathrm{C}\end{array}$ \\
\hline 50 & $5 \mathrm{~cm}$ & $87^{\circ} \mathrm{C}$ & $\begin{array}{l}223^{\circ} \mathrm{C} \\
268^{\circ} \mathrm{C}\end{array}$ \\
\hline 60 & $3 \mathrm{~cm}$ & $92^{\circ} \mathrm{C}$ & $\begin{array}{l}225^{\circ} \mathrm{C} \\
270^{\circ} \mathrm{C}\end{array}$ \\
\hline
\end{tabular}

Table 5.5: Marshal Stability Test for plastic replacement

\begin{tabular}{|c|c|c|}
\hline PLASTIC\% & Stability KN & $\begin{array}{c}\text { Flow value } \\
\text { mm }\end{array}$ \\
\hline Bitumen & 36.64 & 5.5 \\
\hline 10 & 38.68 & 6 \\
\hline 20 & 44.68 & 5 \\
\hline 30 & 57.03 & 4 \\
\hline 40 & 51.93 & 4.6 \\
\hline
\end{tabular}




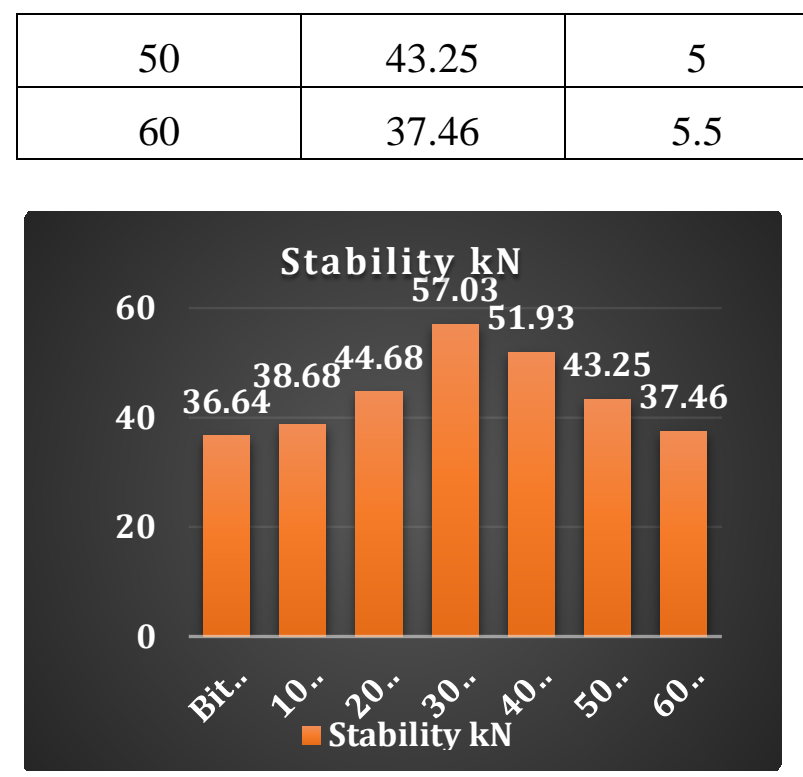

Chart -2: Stability Graph for plastic replacement

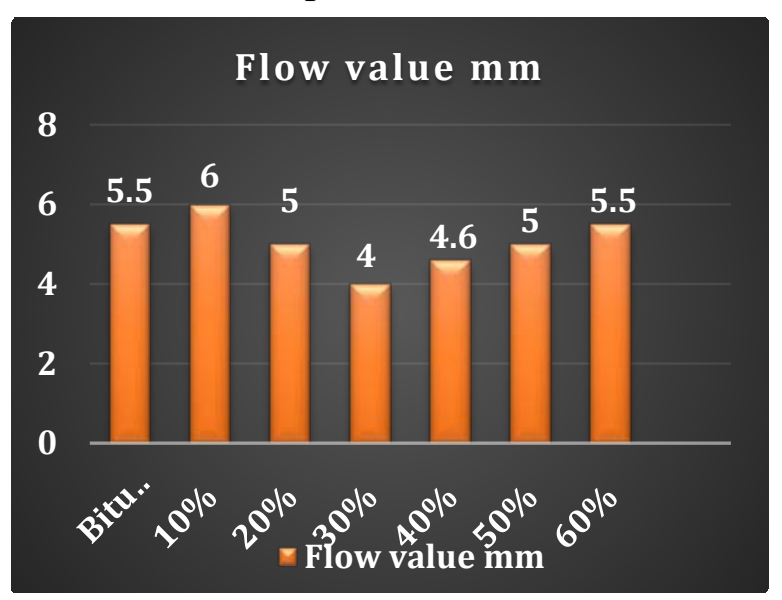

Chart -3: Flow Graph for plastic replacement

\section{b) RUBBER REPLACEMENT}

Rubber is renewal with bitumen in sundry proportions like 10\%, 20\%, 30\%, $40 \%, 50 \%$ \& 60\% (Marshall Stability test, Ductility test, softening point, flash and fire point test) where executed for every replacement of plastic proportions and note down the readings respectively.

Table 5.6: Tests on Rubber Replaced Bitumen

\begin{tabular}{|c|c|l|c|}
\hline $\begin{array}{c}\text { RUBBER } \\
\%\end{array}$ & $\begin{array}{c}\text { Ductility } \\
\mathbf{( c m )}\end{array}$ & $\begin{array}{l}\text { Softening } \\
\text { Point } \\
\left({ }^{\circ} \mathbf{C}\right)\end{array}$ & $\begin{array}{l}\text { Flash } \\
\text { and } \\
\text { Fire } \\
\text { Point }\left({ }^{\circ} \mathbf{C}\right)\end{array}$ \\
\hline Bitumen & $54 \mathrm{~cm}$ & $55^{\circ} \mathrm{C}$ & $\begin{array}{l}180^{\circ} \mathrm{C} \\
210^{\circ} \mathrm{C}\end{array}$ \\
\hline 10 & $13.75 \mathrm{~cm}$ & $58^{\circ} \mathrm{C}$ & $\begin{array}{l}195^{\circ} \mathrm{C} \\
210^{\circ} \mathrm{C}\end{array}$ \\
\hline
\end{tabular}

\begin{tabular}{|c|c|c|c|}
\hline 20 & $9.5 \mathrm{~cm}$ & $60^{\circ} \mathrm{C}$ & $\begin{array}{l}205^{\circ} \mathrm{C} \\
222^{\circ} \mathrm{C}\end{array}$ \\
\hline 30 & $7.75 \mathrm{~cm}$ & $64.5^{\circ} \mathrm{C}$ & $\begin{array}{l}225^{\circ} \mathrm{C} \\
249^{\circ} \mathrm{C}\end{array}$ \\
\hline 40 & $7 \mathrm{~cm}$ & $76^{\circ} \mathrm{C}$ & $\begin{array}{l}230^{\circ} \mathrm{C} \\
252^{\circ} \mathrm{C}\end{array}$ \\
\hline 50 & $5.5 \mathrm{~cm}$ & $80^{\circ} \mathrm{C}$ & $\begin{array}{l}232^{\circ} \mathrm{C} \\
258^{\circ} \mathrm{C}\end{array}$ \\
\hline 60 & $2.5 \mathrm{~cm}$ & $89^{\circ} \mathrm{C}$ & $\begin{array}{l}240^{\circ} \mathrm{C} \\
265^{\circ} \mathrm{C}\end{array}$ \\
\hline
\end{tabular}

Table 5.7: Marshal Stability Test for plastic replacement

\begin{tabular}{|c|c|c|}
\hline RUBBER & Stability KN & Flow value mm \\
\hline Bitumen & 36.64 & 5.5 \\
\hline 10 & 39.19 & 4 \\
\hline 20 & 45.82 & 3.5 \\
\hline 30 & 59.58 & 2.6 \\
\hline 40 & 52.44 & 3 \\
\hline 50 & 44.55 & 3.5 \\
\hline 60 & 37.94 & 5 \\
\hline
\end{tabular}

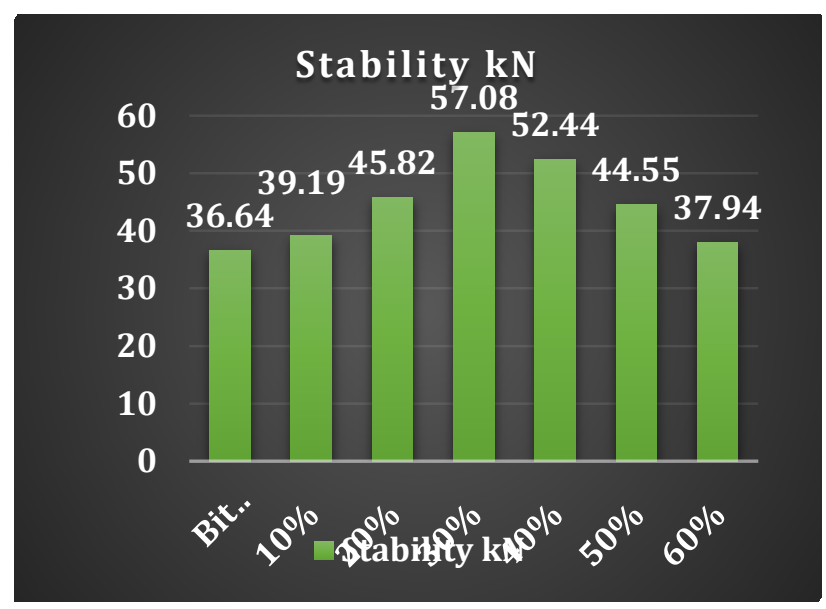

Chart -4: Stability Graph for rubber replacement 


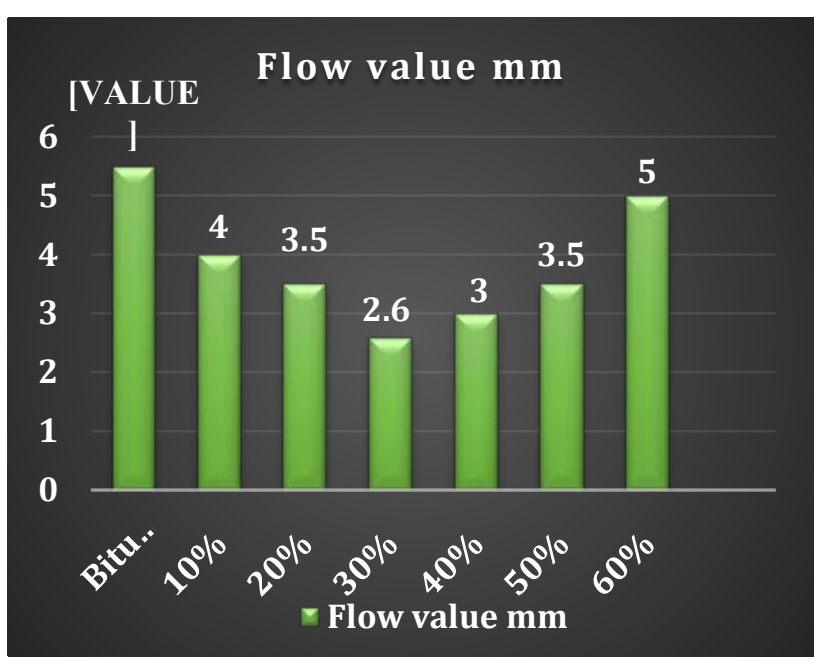

Chart -5: Flow Graph for rubber replacement

\section{c) PLASTIC \& RUBBER}

\section{REPLACEMENT}

Plastic \& Rubber is substituted with bitumen in assorted proportions like 10\%, 20\%, 30\%, $40 \%, 50 \%$ \& $60 \%$ (Marshall Stability test, Ductility test, softening point, flash and fire point test) where executed for every replacement of plastic and rubber proportions and note down the readings respectively.

Table 5.8: Tests on Plastic and Rubber Replaced Bitumen

\begin{tabular}{|c|c|c|c|}
\hline $\begin{array}{c}\text { PLASTIC } \\
\text { \& } \\
\text { RUBBER } \\
\text { \% }\end{array}$ & $\begin{array}{c}\text { Ductility } \\
\text { (cm) }\end{array}$ & $\begin{array}{c}\text { Softening } \\
\text { Point) }\left({ }^{\circ} \mathbf{C}\right)\end{array}$ & $\begin{array}{c}\text { Flash } \\
\text { and } \\
\text { Fire } \\
\text { Point }\left({ }^{\circ} \mathbf{C}\right)\end{array}$ \\
\hline Bitumen & $53 \mathrm{~cm}$ & $53^{\circ} \mathrm{C}$ & $\begin{array}{c}180^{\circ} \mathrm{C} \\
210^{\circ} \mathrm{C}\end{array}$ \\
\hline $5 \mathrm{P}+5 \mathrm{R}$ & $22.5 \mathrm{~cm}$ & $58^{\circ} \mathrm{C}$ & $\begin{array}{c}201^{\circ} \mathrm{C} \\
236^{\circ} \mathrm{C}\end{array}$ \\
\hline $10 \mathrm{P}+10 \mathrm{R}$ & $10.5 \mathrm{~cm}$ & $64.5^{\circ} \mathrm{C}$ & $\begin{array}{c}205^{\circ} \mathrm{C} \\
240^{\circ} \mathrm{C}\end{array}$ \\
\hline $15 \mathrm{P}+15 \mathrm{R}$ & $8.25 \mathrm{~cm}$ & $70^{\circ} \mathrm{C}$ & $\begin{array}{c}218^{\circ} \mathrm{C} \\
256^{\circ} \mathrm{C}\end{array}$ \\
\hline $20 \mathrm{P}+20 \mathrm{R}$ & $5.75 \mathrm{~cm}$ & $80^{\circ} \mathrm{C}$ & $\begin{array}{c}223^{\circ} \mathrm{C} \\
260^{\circ} \mathrm{C}\end{array}$ \\
\hline $25 \mathrm{P}+25 \mathrm{R}$ & $5 \mathrm{~cm}$ & $83.5^{\circ} \mathrm{C}$ & $\begin{array}{c}230^{\circ} \mathrm{C} \\
262^{\circ} \mathrm{C}\end{array}$ \\
\hline $30 \mathrm{P}+30 \mathrm{R}$ & $3 \mathrm{~cm}$ & $85^{\circ} \mathrm{C}$ & $\begin{array}{c}233^{\circ} \mathrm{C} \\
265^{\circ} \mathrm{C}\end{array}$ \\
\hline
\end{tabular}

\begin{tabular}{|c|c|c|}
\hline PLASTIC+RUBBER & $\begin{array}{c}\text { Stability } \\
\text { KN }\end{array}$ & $\begin{array}{c}\text { Flow } \\
\text { value } \\
\text { mm }\end{array}$ \\
\hline Bitumen & 36.64 & 5.5 \\
\hline $5+5$ & 51.93 & 2.5 \\
\hline $10+10$ & 58.57 & 2.3 \\
\hline $15+15$ & 73.34 & 2 \\
\hline $20+20$ & 53.23 & 3.5 \\
\hline $25+25$ & 45.55 & 4.3 \\
\hline $30+30$ & 38.22 & 5 \\
\hline
\end{tabular}

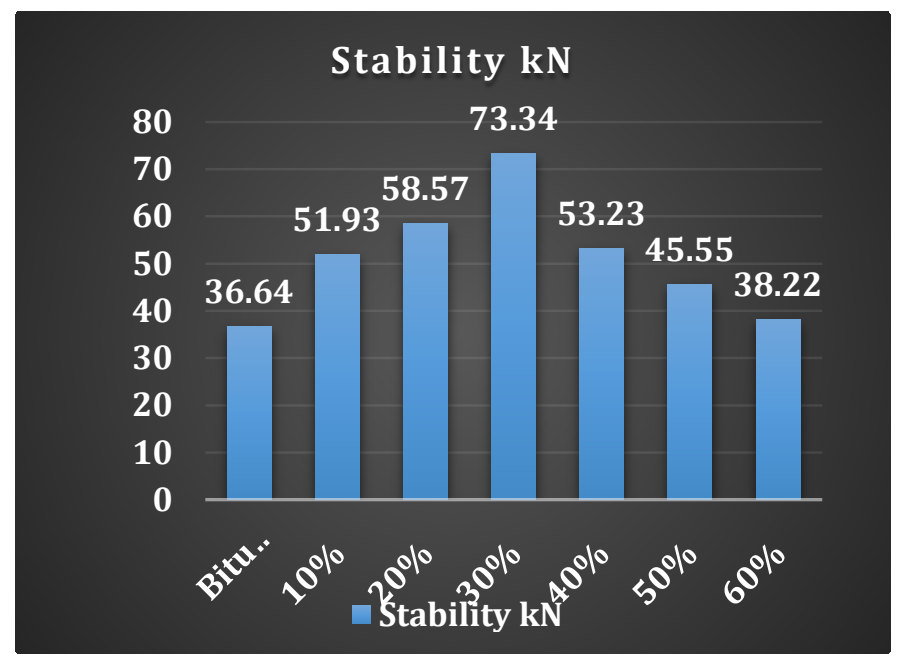

Chart -6: Stability Graph for plastic \& rubber replacement

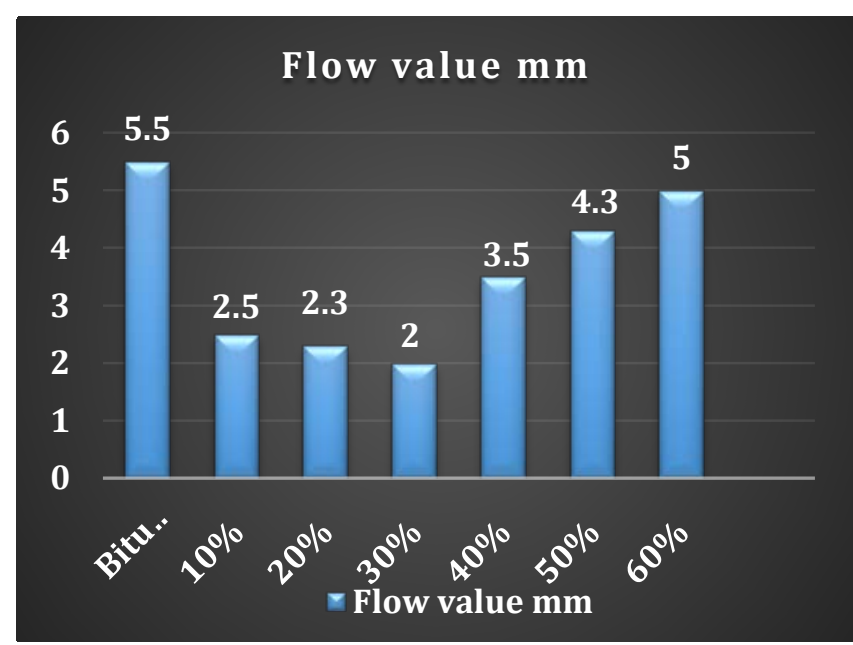

Chart -7: Flow Graph for plastic \& rubber replacement

Table -2: Marshal Stability Test for plastic replacement 


\begin{tabular}{|c|l|c|l|}
\hline $\begin{array}{c}\text { Material } \\
\text { Needed } \\
\text { TY }\end{array}$ & $\begin{array}{l}\text { QUANTI } \\
\text { RATE }\end{array}$ & $\begin{array}{c}\text { TOTA } \\
\text { L } \\
\text { COST }\end{array}$ \\
\hline $\begin{array}{c}\text { Stones(20m } \\
\text { m-6mm) }\end{array}$ & $187.5 \mathrm{~m}^{3}$ & $\begin{array}{c}2500 / \\
\mathrm{m}^{3}\end{array}$ & $\begin{array}{l}\text { Rs }=46 \\
8,750\end{array}$ \\
\hline $\begin{array}{c}\text { Binder(Bitu } \\
\text { men) }\end{array}$ & 11.55 tons & $\begin{array}{l}40000 / \mathrm{t} \\
\text { on }\end{array}$ & $\begin{array}{l}\mathrm{Rs}=46 \\
0,000\end{array}$ \\
\hline Plastic & 4.95 tons & $\begin{array}{c}10000 / \mathrm{t} \\
\text { on }\end{array}$ & $\begin{array}{l}\mathrm{Rs}=49, \\
500\end{array}$ \\
\hline Laying & - & - & $\begin{array}{l}\mathrm{Rs}=30 \\
00\end{array}$ \\
\hline \multicolumn{2}{|c|}{$10 \%$ contractor profit } & $\begin{array}{l}\mathrm{Rs}=97, \\
825\end{array}$ \\
\hline
\end{tabular}

b) Rubber Replacement

$\mathrm{L}=1 \mathrm{~km}, \mathrm{~B}=$ Double lane, $\mathrm{D}=\mathbf{2 5 \mathrm { mm }}$

\begin{tabular}{|c|c|c|l|}
\hline $\begin{array}{c}\text { Material } \\
\text { Needed }\end{array}$ & $\begin{array}{l}\text { QUANTI } \\
\text { TY }\end{array}$ & RATE & $\begin{array}{l}\text { TOTAL } \\
\text { COST }\end{array}$ \\
\hline $\begin{array}{c}\text { Stones(20m } \\
\text { m-6mm) }\end{array}$ & $187.5 \mathrm{~m}^{3}$ & $\begin{array}{c}2500 / \\
\mathrm{m}^{3}\end{array}$ & $\begin{array}{l}\text { Rs }=468, \\
750\end{array}$ \\
\hline $\begin{array}{c}\text { Binder(Bitu } \\
\text { men) }\end{array}$ & 11.55 tons & $\begin{array}{l}40000 / \mathrm{t} \\
\text { on }\end{array}$ & $\begin{array}{l}\mathrm{Rs}=460, \\
000\end{array}$ \\
\hline Rubber & 4.95 tons & $\begin{array}{c}60000 / \mathrm{t} \\
\text { on }\end{array}$ & $\begin{array}{l}\mathrm{Rs}=29,7 \\
00\end{array}$ \\
\hline \multicolumn{2}{|c|}{ Laying } & - & $\mathrm{Rs}=3000$ \\
\hline \multicolumn{2}{|c|}{$10 \%$ contractor profit } & $\begin{array}{l}\mathrm{Rs}=95,8 \\
45\end{array}$ \\
\hline \multicolumn{4}{|l|}{}
\end{tabular}

VI. ESTIMATION AND COMPARISON

7.1 Normal bitumen

$\mathrm{L}=\mathbf{1 k m}, \mathrm{B}=$ Double lane, $\mathrm{D}=\mathbf{5 0} \mathrm{mm}$

\begin{tabular}{|c|c|c|c|}
\hline $\begin{array}{l}\text { Material } \\
\text { Needed }\end{array}$ & $\begin{array}{l}\text { QUA } \\
\text { NTIT } \\
\text { Y }\end{array}$ & RATE & $\begin{array}{c}\text { TOTAL } \\
\text { COST }\end{array}$ \\
\hline $\begin{array}{l}\text { Stones(20m } \\
\text { m-6mm) }\end{array}$ & $375 m^{3}$ & $\begin{array}{c}2500 / \\
\mathrm{m}^{3}\end{array}$ & $\mathrm{Rs}=937,500$ \\
\hline $\begin{array}{l}\text { Binder(Bitu } \\
\text { men) }\end{array}$ & $\begin{array}{l}16.5 \\
\text { tons }\end{array}$ & $\begin{array}{l}40000 / t \\
\text { on }\end{array}$ & $\mathrm{Rs}=660,000$ \\
\hline Laying & - & - & $\mathrm{Rs}=3000$ \\
\hline \multicolumn{3}{|c|}{$10 \%$ contractor profit } & $\mathrm{Rs}=160,050$ \\
\hline
\end{tabular}

\subsection{Replacement Bitumen with Waste}

a) Plastic Replacement

$\mathrm{L}=\mathbf{1 k m}, \mathrm{B}=$ Double lane, $\mathrm{D}=\mathbf{2 5 \mathrm { mm }}$

$$
\text { Total }=\text { Rs 1058,000 }
$$

c) Plastic \& Rubber Replacement

$\mathrm{L}=1 \mathrm{~km}, \mathrm{~B}=$ Double lane, $\mathrm{D}=\mathbf{2 5 \mathrm { mm }}$

\begin{tabular}{|c|l|c|l|}
\hline $\begin{array}{c}\text { Material } \\
\text { Needed }\end{array}$ & $\begin{array}{l}\text { QUANTI } \\
\text { TY }\end{array}$ & RATE & $\begin{array}{l}\text { TOTAL } \\
\text { COST }\end{array}$ \\
\hline $\begin{array}{c}\text { Stones(20m } \\
\text { m-6mm) }\end{array}$ & $187.5 \mathrm{~m}^{3}$ & $\begin{array}{c}2500 / \\
\mathrm{m}^{3}\end{array}$ & $\begin{array}{l}\text { Rs }=468, \\
750\end{array}$ \\
\hline $\begin{array}{c}\text { Binder(Bitu } \\
\text { men) }\end{array}$ & 11.55 tons & $\begin{array}{c}40000 / \mathrm{t} \\
\text { on }\end{array}$ & $\begin{array}{l}\mathrm{Rs}=460, \\
000\end{array}$ \\
\hline Plastic & 2.475 tons & $\begin{array}{c}10000 / \mathrm{t} \\
\text { on }\end{array}$ & $\begin{array}{l}\mathrm{Rs}=24,7 \\
50\end{array}$ \\
\hline Rubber & 2.475 tons & $\begin{array}{c}60000 / \mathrm{t} \\
\text { on }\end{array}$ & $\begin{array}{l}\mathrm{Rs}=14,8 \\
50\end{array}$ \\
\hline \multicolumn{2}{|c|}{-} & - & $\mathrm{Rs}=3000$ \\
\hline Laying & & $\begin{array}{l}\mathrm{Rs}=97,1 \\
35\end{array}$ \\
\hline \multicolumn{2}{|c|}{$10 \%$ contractor profit } &
\end{tabular}

Total $=$ Rs 1068,500

Total $=$ Rs 1080,000 


\section{COST COMPARISON}

\begin{tabular}{|l|c|c|c|c|}
\hline \multicolumn{1}{|c|}{ Material Needed } & $\begin{array}{c}\text { Plain } \\
\text { Bitumen } \\
\text { Process }\end{array}$ & $\begin{array}{c}\text { Plastic- } \\
\text { Bitumen road }\end{array}$ & $\begin{array}{c}\text { Rubber- } \\
\text { Bitumen road }\end{array}$ & $\begin{array}{c}\text { Plastic \& Rubber- } \\
\text { Bitumen road }\end{array}$ \\
\hline \multicolumn{1}{|c|}{ Stones(20mm-6mm) } & $375 \mathrm{~m}^{3}$ & $187.5 \mathrm{~m}^{3}$ & $187.5 \mathrm{~m}^{3}$ & $187.5 \mathrm{~m}^{3}$ \\
\hline $\mathbf{5 0 / 7 0}$ Bitumen & 16.5 tons & 11.55 tons & 11.55 tons & 11.55 tons \\
\hline Waste & ---------- & 4.95 tons & 4.95 tons & 2.475 tons +2.475 tons \\
\hline \multicolumn{1}{|c|}{ Cost Rs. } & $\mathbf{1 7 6 1 , 0 0 0}$ & $\mathbf{1 0 8 0 , 0 0 0}$ & $\mathbf{1 0 5 8 , 0 0 0}$ & $\mathbf{1 0 6 8 , 5 0 0}$ \\
\hline Cost Reduced Rs. & NIL & 681000 & 703000 & 692500 \\
\hline Cost Reduction in \% & $\mathbf{0 \%}$ & $\mathbf{3 8 . 5 \%}$ & $\mathbf{4 0 \%}$ & $\mathbf{3 9 . 3 \%}$ \\
\hline Stability kN & 36.64 & 57.03 & 59.58 & 2 times \\
\hline Stability Increased By & 0 times & 1.55 times & 1.62 times & 0.36 times \\
\hline Flow Value Decreased By & 0 times & 0.72 times & 0.47 times & \\
\hline
\end{tabular}

\section{CONCLUSIONS}

1. The graphs describe about assorted content of bitumen and properties of behavior of marshal stability molds, shows positive outcome at $30 \%$ of waste content. Hence we can conclude that among $10 \%, 20 \%, 30 \% 40 \%, 50 \%$ and $60 \% .30 \%$ is the optimum.

2. The bitumen modifier can increase the stability and density of the mix which is suggested for the surface course.

3. It is invent that, the stability is increased 1.55, 1.62 and 2times (plastic, rubber, plastic and rubber respectively) when juxtapose to the normal bituminous mix by restoring waste substances.

4. We researched that, the flow value is decreased 0.72, 0.47 and 0.36 times (plastic, rubber, plastic and rubber respectively), when collate to the normal bituminous mix i.e. with replacing waste materials.

5. The thickness of the pavement layer may be decline to half of normal thickness which results in economy of cost by reducing quantity of aggregate, bitumen and by achieving the work in less duration of time with better quality.
6. Due to use of waste stuff the cost reduction is up to $38.5 \%, 40 \%$ and 39.3 $\%$ ( plastic, rubber and plastic and rubber respectively). When compare to ordinary bitumen.

\section{PURVIEW OF FURTHER STUDIES}

1. This research can be executed for other grades of bitumen.

2. Other waste materials like LDPE, HDPE, fibers, glass powder etc. can be taken for the substitution with bitumen.

\section{REFERENCES}

1. Tariq Shehab and Ida Meisami-Fard "Cost-Estimating Model for Rubberized Asphalt Pavement RehabilitationProjects". DOI: 10.1061/(ASCE)IS.1943-

555X.0000146. @ 2013 American Society of Civil Engineers.

2. Fernando Moreno Navarro and $M$. Carmen Rubio Gámez "Influence of Crumb Rubber on the Indirect Tensile Strength and Stiffness Modulus of Hot Bituminous Mixes". DOI: 10.1061/(ASCE)MT.1943-5533.0000436. 
(C) 2012 American Society of Civil Engineers.

3. Hyun Hwan Kim, Kyu-Dong Jeong, Moon Sup Lee, and Soon-Jae Lee "Effect of FT Paraffin Wax Contents on Performance Properties of Crumb Rubber-Modified Asphalt Binders" DOI: 10.1061/(ASCE)MT.1943-5533.0001267.

(C) 2015 American Society of Civil Engineers.

4. Mahabir panda and Mayajit Mazumdar "Utilization of Reclaimed Polyethylene in Bituminous Paving Mixes" DOI:10.1061/(ASCE)08991561(2002)146 (527)

5. S. Rajesekaran, Dr. R. Vasudevan, Dr. Samuvel Paulraj "Reuse of Waste Plastic Coated Aggregate-Bitumen Mix Composite for Road Application-Green method "American Journal of Engineering Research (AJER)eISSN:2320-0847, p-ISSN: 2320-0936 Volume 2, Issue 11.

6. Dr. Malik Shoeb Ahmad 'Low Density Polyethylene Modified Dense Graded Bituminous Macadam" International Journal of Engineering Trends and Technology(IJETT)-Volume 16 Number 8-Oct 20142320-0936

7. Mr.P.B. Rajmane, Prof. A.K. Gupta, Prof.D.B. Desai. Desai. Desai. Desai. Desai "Effective Use of Waste Plastic in Construction of Flexible Pavement for Improving the Performance" IOSR Journal of Mechanical and Civil Engineering (IOSR-JMCE) ISSN:22781684.

8. Logeshkumaran, "Experimental Study On Partial Replacement of Bitumen with Lignin and Plastic" (2018), Volume 5, Issue -4, ISSN 2393-8374.

22. e. MORTH 500
9. Syed Nasir, Muhammad Ayoub, Ahmed Bilal, Badar Amjad. Ehsanullah kakar "Effective use of Waste Plastic as Bitumen Strength Modifier "Civil Engineering and Architecture2 (9);313.313,2014.

10. Anzar Hamid Mir, "Use of Plastic Waste in Pavement Construction", ISSN (e):2250-3021, ISSN (p):22788719, IOSR.

11. Huang, B., Mohammad, L. N., Graves, P. S., and Abadie, C. "Louisiana experience with crumb rubber-modified hot-mix asphalt pavement." Transportation Research Record 1789, Transportation Research Board, Washington, DC, 1-13. (2002).

12. Sherwood, P. T. (1995). "Alternative materials in road construction". Thomas Telford Publications, London, 1995

13. L. Flynn, "Recycled Plastic finds it Home in Asphalt Binder", Roads and Bridges (1993).

14. Plastics for Environment and Sustainable Development, ICPE, 8(1) (2007).

15. Yash Menaria, Rupal Sankhla. "Use of Waste Plastic in Flexible PavementsGreen Roads" published 9 September 2015 Copyright (C) 2015 by authors and Scientific Research Publishing Inc.

16. Justo, C.E.G. and Veeraragavan, A. "Utilization of Waste Plastic Bags in Bituminous Mix for Improved Performance of Roads". Banglore University, Bengaluru. (2002)
17. CODE BOOKS
18. a. IS 2386:1963
19. b. IS 73:2013
20. c. IRC 37:2012
21. d. SP 20 